International Journal of Soft Computing 14 (1): 6-10, 2019

ISSN: $1816-9503$

(C) Medwell Journals, 2019

\title{
Rendering a Video on the Image Coordinates Using Augmented Reality
}

\author{
${ }^{1} \mathrm{P}$. Mahalakshmi, ${ }^{2}$ Sri Harshavardhan Pathuri and ${ }^{2} \mathrm{~K}$. Sampath Kumar \\ ${ }^{1}$ Department of CSE, \\ ${ }^{2}$ Department of Computer Science, SRM University, Chennai, India
}

\begin{abstract}
Augmented reality is the advanced approach of indirect view of the real world environment. It is used to generate sensory inputs like audios, videos and graphics in reval time. Augmented reality can also be used for rendering the videos on a background and play them with in the image coordinates. This concept of augmented reality is simply to emphasize on image recognition and then combining the real time visuals with the help of the advanced approach of augmented reality technology. Augmented reality brings out the components of the digital world into a real world sensation. In this study, we present an android application with the concept of augmented reality which helps in scanning the images and plays the mapped videos that match with the image. Initially, the application requires permissions from the user to access the device camera for scanning the images. This acts as input for the application. As soon as the camera recognizes the images it compares the pixels and coordinates with the predefined images markers present in the database. Once the marker is recognized it will fetch the linked video and plays it. This video will be played only on the image coordinates without disturbing the surrounding content. When the camera is closer or when it is taken away from the image the video player gets resized itself according to the image coordinates. Finally, we validate our research to render the video on the image.
\end{abstract}

Key words: Augmented reality, image processing, video rendering, AR tool kit, augmented, pixels

\section{INTRODUCTION}

Augmented reality is an advanced technology for superimposing the enhanced content on an image. Augmented reality is the advanced approach of indirect view of the real world environment (Rohmer et al., 2015). It is used to generate sensory inputs like audios, videos and graphics in real time. Augmented reality can also be used for rendering the videos on a background and play them with in the image coordinates. This concept of augmented reality is simply to emphasize on image recognition and then combining the real time visuals (Cho et al., 2016) with the help of the advanced approach of augmented reality technology. In this study, we present an android application that provides a platform which helps in playing the related videos (Fig. 1-4) by recognizing the image (Fig. 3). This application takes the image as an input. As shortly as the camera recognizes the image it matches with the pre-defined image markers present in the database. Once the marker is recognized it will fetch, the predefined video and it keeps the virtual player over the image and start playing the video. When the camera is closer to the image or far away from the image maker, the video player size will also keep on changing, eventually this will clear the entire procedure will come realistic in actual time. This application basically uses AR toolkit which takes the image as an input. This

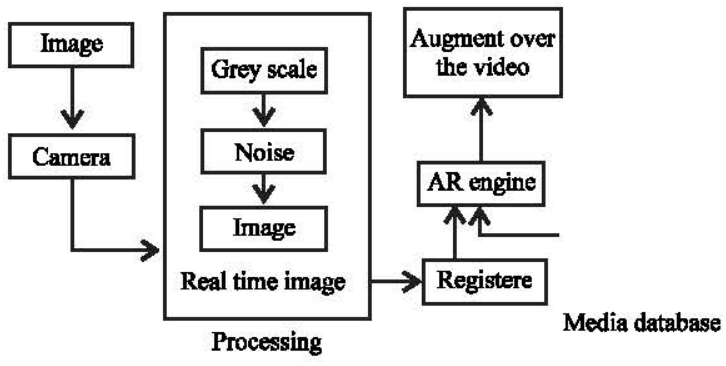

Fig. 1: Architecture diagram describing the process

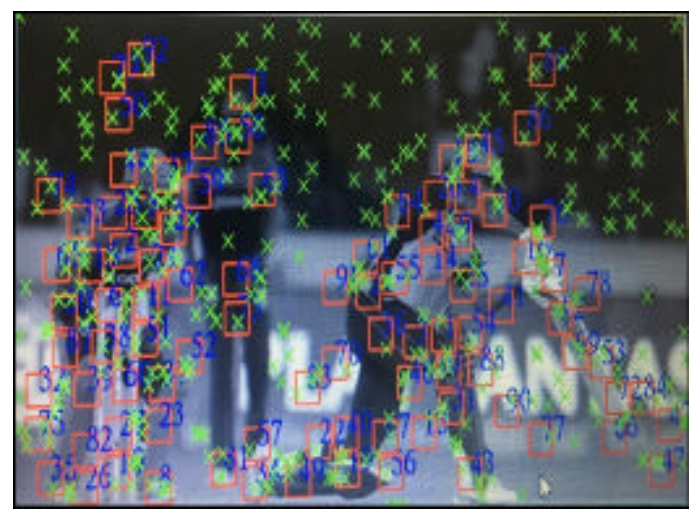

Fig. 2: Sample image markers made with AR toolkit for image recognition 


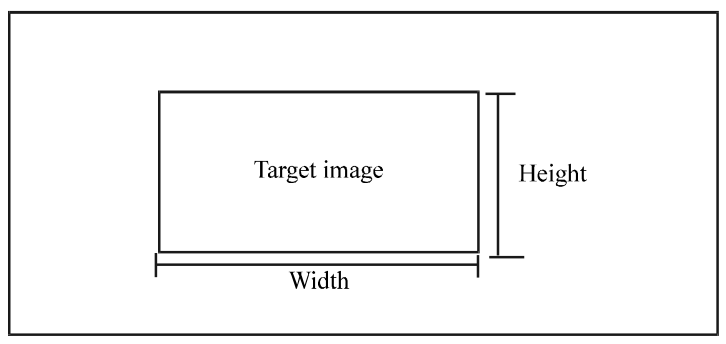

Fig. 3: Target image

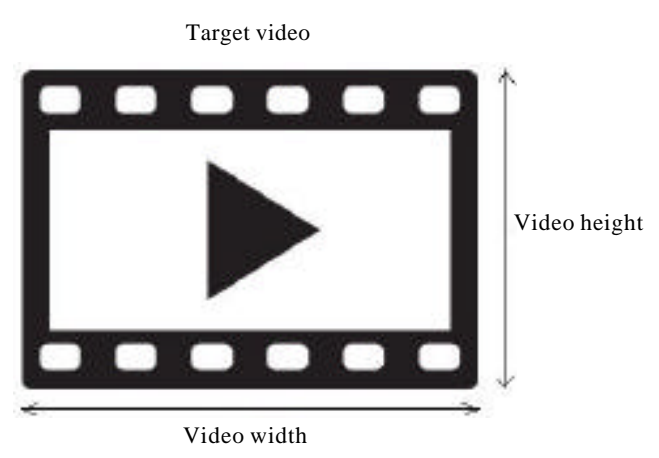

Fig. 4: Video to be played on target image

process includes the extraction of feature set files and information set (iset) files with the help of AR toolkit. These files are linked to the corresponding video. When the user tries to scan the image these saved feature sets are matched with the input image. When the images get matched the linked video will be played on that particular image coordinates.

\section{Literature review}

Live texturing of characters: Augmenting the 3D images on coloured images from the drawing book (Magnenat et al., 2015) is the best example of augmented reality application. Now a days children are much attracted to the digital gadgets like mobile phones tablets and computers. They are very much interested in playing games in mobile phones and tablets rather than painting and other outdoor activities. Present generation uses painting applications from these mobile devises to show their creativity. So, we need an application which should help in presenting the children work more creatively and impressively, so that, they will show interest in activities like painting. This study helps in presenting an augmented reality painting book application. This application can be used on the images given in their painting book. Children has to colour the empty outline of the characters like animals and cartoon images in the drawing book. After showing their creativity in colouring the characters they need to scan the coloured images or characters using the augmented reality application. The application captures the image colours and presents a 3-D Version of the image filled with the colours same as in the image. This type of presenting their work creates more fun to the children and they shows more interest in colouring more images and improving their creativity. Thus, this application helps the children to be more creative and enjoy their creative work with the augmented video of their creation in the painting book.

Content authoring: Content authoring (Cho et al., 2016) can also be done with the help of the augmented reality. Scanning the materialistic objects like buildings from the real world and getting their information is the main aim of the content authoring. This helps the users to have a better ocular experience. This also, helps in guiding the users in the new places by displaying the buildings information. This study proposes a simple content authoring methodology. Here, the buildings are scanned and set some coordinates to present the visual information about the building. The buildings are scanned for the vectors in the $2 \mathrm{D}$ plane to augment the information of the building on the plane. The buildings information and the unique feature points are generated previously and are maintained in the database. When the user scans for the building information the applications try to fetch the building feature points to get the linked information in the database. When the feature points mapped in the $2 \mathrm{D}$ plane are found the information of the building is rendered on the 2D plane of the building. Finally, the users can know the buildings information easily even in new places. This also serves a great visual experience to the users. This technique of augmented reality is the advanced approach of content authoring.

Photorealistic augmented reality: Photorealistic augmented reality has some limitation in photorealistic approach (Rohmer et al., 2015). This is because of the lack of processing power in the mobile devices. Photorealistic work is presenting the materialistic work same as it is by using the media like painting drawing, etc. This gives the user a realistic presence of the object. To present this research in augmented reality there are many limitations like processing speed of the mobile devices the network speed and soon. To overcome this limitations the researchers in this study proposed a pinion method which superimposes the virtual objects in the mobile devices. This approach uses computer graphics and virtual reality in augmented reality. This approach needs a setup which helps in the optical tracking of the objects placed. This setup includes the high definition cameras which captures 
the perfect images with high definition. This setup also requires a fisheye lenses so, that, the whole region of experiment is clearly visible. The whole setup is connected to a stationary PC. The virtual objects rendered in the mobile devices uses direct indirect and shadow light of the objects which serves for the most accuracy of the virtual object. Thus the virtual objects created are same like the original objects. These virtual objects are rendered as the original objects which helps the augmentation in the photorealistic way. The rendered objects with the help of the light source is same as the original objects captured. Thus photorealistic approach of augmentation can be done with this setup using the cameras and a stationary $\mathrm{PC}$.

Driver assistant system: Driver face detection (Guo et al., 2006) is also made possible with the use of augmented reality. Face detection generally mean the identification of faces and estimating the heads position of the person. This serves a great purpose for the drivers of vehicles. This acts as the driver's virtual assistance with fast responses. For this to exist in the present world it must be a fast design and must have much accuracy in matching the faces. The algorithm in this approach is proposed in this study. The first step in this process is the identification is the face detection. After the detection of the face several scales are applied to get the maximum possibilities. This process also uses SVMs classifiers. Bilateral-projection matrix is used for the face pose estimation. The process flows as the face detection, tracking the detected face and finally estimating the head position with the help of bilateral-projection matrix principle component analysis. The detected face is verified by matching with the existing database with several application of scales to the detected face. If the detection is not success if reads the next frame in the database. And this process ends when all the frames are processed and no more frame is available for matching. This eventually mean the face cannot be detected. So, it tries for the next face for detection. If the detected face is matched with any one of the frames then it seems success and tries for the tracking of the face position. If tracking is also success then tries for the estimation of the heads position. If it is not success again it tries for the next frame and process continuous till it success or fails. Thus, estimation of the face position is made possible with help of the augmented reality. This serves a great purpose for the driver's assistance.

LabView based augmented reality: LabVIEW (Migliore et al., 2016) is also a best augmented reality smartphone application. This application helps to augment the LabVIEW in the mobile devices. This application uses adobe AIR for presenting the same code into the application which is the major technology in the present day applications. LabVIEW which is the abbreviated form of laboratory virtual instrument engineering work bench which is used for the better graphical representation in the major coding languages. This LabVIEW approach of augmented reality basically follows client server model. For this the application uses adobe air for the client server model. This application also uses pattern recognition technique for the augmentation in the smartphones. The client captures the images from the surroundings using the mobile devices. The captured images are sent for the pattern recognition which is the major step in the process of augmentation. The client has three modules like TCP/IP which is an active internet source like wifi or lan, camera module and the augmented module. Here, the camera acts as the input and the augmented module is the output of the whole process. After the pattern recognition is processed successfully an enhanced content is rendered on the camera input. The pattern recognition process is done in the server part and sends the output to the mobile devices.

\section{MATERIALS AND METHODS}

Problem formulation: Our method of playing an AR movie on an image updates the related video with in the image coordinates. Scanning the image from the real-time either from the newspaper or the digital images is a bit easy task with the help of the camera function but matching with images with our database and recognizing them is a bit challenging task. So, we divide the input into frames and each frame is matched with the existing database. The input image markers are matched with the existing image markers. Basic corner detection is used for implementing the markers (Fig. 2) on the image. For getting the image recognized we need to train an image, so, that we can add the corresponding video path, so, that the video corresponding to the path will be played. So, for training the image we use AR toolkit for extracting the feature set of the images. These feature sets of the images will be saved in the database and a video path will be linked which need to be played when the image is recognized. AR (Augmented Reality) toolkit is generally the basic tool kit which helps in extracting the feature set of the images which helps in the augmented reality processing. This uses open CV (Computer Vision) library which is widely used for image processing (Fig. 2). These files are saved as the iset and fset extensions in the database which helps in the further process. While playing the video when we come close to the image the 


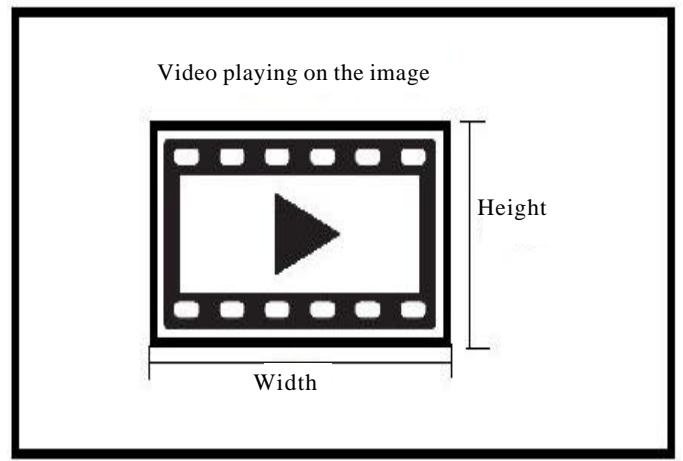

Fig. 5: Video playing on target image co-ordinates

image becomes larger and our video coordinates should also increase, according to the coordinates, the same should happen when we go far away from the image. The renderer and the movie controller will help in accomplishing this task. Finally, we can play the movie or the related video with in the image coordinates (Fig. 5). without disturbing the surroundings. Finally, the user gets the video of the image in a simple way with the use of this simple AR application.

\section{RESULTS AND DISCUSSION}

\section{Implementation}

Camera surface: This is the first and foremost step in the implementation part. The input for the whole process is an image input which helps in playing the video related to image as the output. So, we use the camera for scanning the input. As soon as the application launches the camera of the mobile device is accessed with the permission of the user and keeps on scanning the images. This process basically gives two outputs. One helps in the displaying part which is displayed on the mobile screen of the user. The other goes to the next part which is recognizing part which helps in matching to the database to play the respective video.

Real time image processing: The real time image processing Fig. 1 is always in high demand for many applications used in security system, remote sensing and manufacturing process and multimedia those require to have high performance. This is basically used to extract the video from the database. In this architecture of real image processing it consists.

Grey scale, noise and image: Grey scale images is also known as black-and-white are composed exclusively of shades of grey, varying from black at the weakest intensity to white at the strongest. So, in our architecture this is used of recognizing the type of image frames of the video either colour or black and white. Noise Fig. 1 in image processing is basically a random variation of brightness and shadows of the image that disturbs the pixel clarity of the image. It is an undesirable by-product of image capture. So, here this part is used for recognizing the clarity of image frame of the video.

Recognizing: This process gets the input the camera surface which is an image scanned with the help of the camera. The recognizing part matches the incoming input images with the existing trained images in the database. The training of images is done with the help of the AR toolkit which extracts the unique feature dataset from the images. These datasets are saved locally in the database which helps in the matching purpose. Now the incoming image is matched with the existing datasets of images in the database. When the particular image gets recognized the movie controller will be triggered (Fig. 2).

Render: Our main aim is to play the video (Fig. 4) with in the target image (Fig. 3) coordinates without disturbing the surrounding media. This part is maintained by the render which helps the video to be played with in the coordinates (Fig. 5). When the mobile zooms in the image the video player size must be increased according to the image coordinated. The same thing must also, happen when we zoom out the image the video player size must shrink according to the image coordinates:

$$
\begin{aligned}
& \text { Height } 1 \text { = Height of the target image } \\
& \text { Width } 1 \text { = Width of the target image } \\
& \text { Height } 2 \text { = Height of the video } \\
& \text { Width } 2 \text { = Width of the video } \\
& \text { Height } 3=\text { Height } 2 * \text { (Heigh } 1 \text { ratio) } \\
& \text { Width } 3=\text { Width } 2 *(\text { Width } 1 \text { ratio) }
\end{aligned}
$$

The height of the target image Eq. 1 and the width of the target image is taken. The height and width of the video to be played is also considered (Eq. 3 and 4). New coordinates of the video player is calculated with respect to the image coordinates ratio (Eq. 3 and 4). The render will handle the above situation by getting the image coordinates Eq. 1 and 2 and fixes the unique feature points in the image which are saved in database. This data 
is saved with the help of the AR toolkit which we discussed earlier. The render access these feature set files and get the unique feature points of the image and process the video player rendering on the particular image coordinates. Thus we are able to play the video particularly on the image coordinates (Eq. 3 and 4) without disturbing the surrounding environment.

Movie controller: This event is triggered when the input image gets recognized and matched with the image dataset existing in the database. If the event happens successfully a particular video has to play which is given by the application admin. Either the video path will be present or the video itself will be present in the database. Movie controller helps in getting the video and play according to the instructions given by the admin. Movie controller acts as a platform or the media player for the requested video. Movie controller will help in playing the video on the image where the coordinates are set by the render.

\section{CONCLUSION}

In this study, we present the related video to be played when it finds the particular trained image which is present in our database. We generally maintain the data set in our device storage and the video will be triggered to play on the image. This needs the regular update of the application to avail the new videos. So, to overcome this challenge we need to save the image dataset also on the server, so that, it doesn't require regular updates. With this the app works totally online and a bit challenging task to load the whole data from the servers. But this process helps in working for the regular newspaper to lend the news more creatively and innovatively.

\section{REFERENCES}

Cho, N.H., Q. Wu, J. Xu and J. Zhang, 2016. Content authoring using single image in urban environments for augmented reality. Proceedings of the 2016 International Conference on Digital Image Computing: Techniques and Applications(DICTA), November 30-December 2, 2016, IEEE, Gold Coast, QLD, Australia, ISBN:978-1-5090-2897-9, pp: 1-7.

Guo, Z., H. Liu, Q. Wang and J. Yang, 2006. A fast algorithm face detection and head pose estimation for driver assistant system. Proceedings of the 8th International Conference on Signal Processing, Volume 3, November 16-20, 2006, Beijing, China pp $1-10$.

Magnenat, S., D.T. Ngo, R.W. Sumner, F. Zund and M. Ryffel et al., 2015. Live texturing of augmented reality characters from colored drawings. IEEE. Trans. Visual. Comput. Graphics, 21 : 1201-1210.

Migliore, E.G., J.Z. Abad and F.Q. Palomeque, 2016. An approach to develop a LabVIEW based augmented reality application for smartphones. Proceedings of the IECON 2016 42nd Annual International Conference on IEEE Industrial Electronics Society, October 23-26, 2016, IEEE, Florence, Italy, ISBN:978-1-5090-3475-8, pp: 4970-4975.

Rohmer, K., W. Buschel, R. Dachselt and T. Grosch, 2015. Interactive near-field illumination for photorealistic augmented reality with varying materials on mobile devices. IEEE. Trans. Visual. Comput. Graphics, 21 : 1349-1362. 